Bull. Korean Math. Soc. 48 (2011), No. 6, pp. 1245-1252

http://dx.doi.org/10.4134/BKMS.2011.48.6.1245

\title{
THE STRUCTURE OF THE REGULAR LEVEL SETS
}

\author{
SEungsu HWANG
}

\begin{abstract}
Consider the $L^{2}$-adjoint $s_{g}^{\prime *}$ of the linearization of the scalar curvature $s_{g}$. If $\operatorname{ker} s_{g}^{\prime *} \neq 0$ on an $n$-dimensional compact manifold, it is well known that the scalar curvature $s_{g}$ is a non-negative constant. In this paper, we study the structure of the level set $\varphi^{-1}(0)$ and find the behavior of Ricci tensor when $\operatorname{ker} s_{g}^{*} \neq 0$ with $s_{g}>0$. Also for a nontrivial solution $(g, f)$ of $z=s_{g}^{* *}(f)$ on an $n$-dimensional compact manifold, we analyze the structure of the regular level set $f^{-1}(-1)$. These results give a good understanding of the given manifolds.
\end{abstract}

\section{Introduction}

Let $M$ be an $n$-dimensional compact manifold and $\mathcal{M}_{1}$ the set of all smooth Riemannian structures on $M$ of volume 1 . The scalar curvature $s_{g}$ is a nonlinear function of the metric $g$. Its linearization at $g$ in the direction of the symmetric two-tensor $h$ is given by

$$
s_{g}^{\prime}(h)=-\Delta_{g} \operatorname{tr} h+\delta_{g} \delta_{g} h-g\left(h, r_{g}\right) .
$$

Here, $\Delta_{g}$ is the negative Laplacian of $g, r_{g}$ is its Ricci tensor, and $\delta_{g}$ is the metric dual of the map on the bundle of symmetric tensors induced by covariant differentiation. The $L^{2}$-adjoint operator $s_{g}^{*}$ of $s_{g}^{\prime}$ with respect to the canonical $L^{2}$-inner product given by $\langle\rangle=,\int_{M} g(,) d v_{g}$ is

$$
s_{g}^{*}(f)=D_{g} d f-g \Delta_{g} f-f r_{g} .
$$

If $\operatorname{ker} s_{g}^{*} \neq 0, s_{g}$ is known to be a non-negative constant. More specifically,

Theorem 1.1 ([5], [6]). If ker $s_{g}^{*} \neq 0$, either $(M, g)$ is Ricci-flat and ker $s_{g}^{* *}=$ $\mathbb{R} \cdot 1$, or $s_{g}$ is a positive constant and ker $s_{g}^{*} \subset \operatorname{ker}\left(\triangle_{g}-\frac{s_{g}}{n-1}\right)$.

Based on this result, Fisher-Marsden suggested the conjecture which states that if $\operatorname{ker} s_{g}^{*} \neq 0$ for a Riemmanian metric $g$ with $s_{g}>0$, then $(M, g)$ is a standard sphere. It turns out by [10] and [11] that there are counter-examples of

Received July 14, 2010; Revised November 3, 2010.

2010 Mathematics Subject Classification. Primary 53C25.

Key words and phrases. scalar curvature, the regular level set, the traceless Ricci tensor.

This Research was supported by the Chung-Ang University Research Grants in 2009.

(C)2011 The Korean Mathematical Society 
Fisher-Marsden conjecture. For example, a finite quotient of a warped product $\left(S^{1} \times_{\phi^{2}} S^{n-1}, d t^{2}+\phi^{2}(t) g_{0}\right)$ satisfies $\operatorname{ker} s_{g}^{\prime *} \neq 0$.

On each connected component of the level set $\varphi^{-1}(0)$, the normal vector field $N_{\varphi}=d \varphi /|d \varphi|$ to $\varphi^{-1}(0)$ is well defined, which will be shown in the next section. Let $z$ be the traceless Ricci tensor given by $z=r_{g}-\frac{s_{g}}{n} g$. From the observation of the structures of the counter-examples mentioned above, we have the following characterization of the structure of the level set $\varphi^{-1}(0)$.

Theorem 1.2. Let $\varphi$ be a non-trivial function in $\operatorname{ker} s_{g}^{*}$ on an $n$-dimensional compact manifold $M$. Then, on each connected component of the level set $\varphi^{-1}(0)$ with the normal vector field $N_{\varphi}=d \varphi /|d \varphi|$,

$$
z\left(X, N_{\varphi}\right)=0
$$

for any tangent vector $X$ to $\varphi^{-1}(0)$. Moreover,

$$
z\left(N_{\varphi}, N_{\varphi}\right) \leq 0
$$

on some connected component of $\varphi^{-1}(0)$.

On the other hand, we consider the total scalar curvature of $M$ defined by

$$
\mathcal{S}(g)=\int_{M} s_{g} d v_{g}
$$

for $g \in \mathcal{C}$, a subset of $\mathcal{M}_{1}$, consisting of constant scalar curvature metrics. Then the Euler-Lagrange equation of $\mathcal{S}$ restricted to $\mathcal{C}$ is given by

$$
z_{g}=s_{g}^{*}(f),
$$

where $z_{g}$ is the traceless Ricci tensor and $f$ is a function on $M$ with vanishing mean value; c.f. [3]. It has been conjectured that a non-trivial solution $(M, g, f)$ of (4) is isometric to a standard $n$-sphere. Analysis of the structure of the components of $f^{-1}(-1)$, as in the case of $\varphi^{-1}(0)$ for $\varphi \in \operatorname{ker} s_{g}^{*}$, gives a good understanding of the given manifold $(M, g)$.

On each connected component of the regular level set $f^{-1}(-1)$, the normal vector field $N_{f}=d f /|d f|$ to $f^{-1}(-1)$ is well defined. In the following, we have a characterization of the level set $f^{-1}(-1)$.

Theorem 1.3. Let $(g, f)$ be a non-trivial solution of (4) on an $n$-dimensional compact manifold $M$. Then, on each connected component of the regular level set $f^{-1}(-1)$ with the normal vector field $N_{f}=d f /|d f|$,

$$
z\left(X, N_{f}\right)=0
$$

for any tanget vector $X$ to $f^{-1}(-1)$. Moreover,

$$
z\left(N_{f}, N_{f}\right) \leq 0
$$

on some connected component of $f^{-1}(-1)$. 


\section{Proofs of Theorems $\mathbf{1 . 2}$ and $\mathbf{1 . 3}$}

There are some similarities between the proofs of Theorems 1.2 and 1.3. We first prove Theorem 1.2.

The differential operator $d^{D}$ of $C^{\infty}\left(S^{2} M\right)$ into $\Lambda^{2} M \otimes T^{*} M$ is defined as

$$
d^{D} h(X, Y, Z)=\left(D_{X} h\right)(Y, Z)-\left(D_{Y} h\right)(X, Z) .
$$

Let $\varphi$ be a non-trivial function in $\operatorname{ker} s_{g}^{\prime *}$. Then $\varphi$ satisfies the following equation

$$
\varphi z=D d \varphi+\frac{s}{n(n-1)} \varphi g .
$$

Let $\Gamma=\varphi^{-1}(0)$. It is known that there are no critical points of $\varphi$ on $\Gamma$; c.f. [6]. Therefore, $\Gamma$ is a union of hypersurfaces of $M$. Note that $|d \varphi|$ is constant on $\Gamma$, since by $(7)$

$$
X\left(|d \varphi|^{2}\right)=2\left\langle D_{X} d \varphi, d \varphi\right\rangle=0
$$

for any tangent vector $X$ to $\Gamma$. Thus a normal vector field $N_{\varphi}=d \varphi /|d \varphi|$ is defined on all of $\Gamma$. Here $\Gamma$ is totally geodesic, since $D_{g} d \varphi=0$ on $\Gamma$. First we need the following lemma.

Lemma 2.1. For $p \in \Gamma$ and a tangent vector $X \in T_{p} \Gamma$ which is orthogonal to $\nabla \varphi$, we obtain

$$
z(X, \nabla \varphi)=0 .
$$

Proof. By Ricci identity (see, for example, [11]),

$$
d^{D} D d \varphi(X, Y, Z)=R(X, Y, Z, \nabla \varphi)
$$

Thus, from the equation (1) with $\varphi$,

$$
\begin{aligned}
0 & =\left(d^{D} s_{g}^{\prime *} \varphi\right)(X, Y, Z) \\
& =R(X, Y, Z, \nabla \varphi)+\left(\frac{s}{n-1} d \varphi \wedge g-d \varphi \wedge r-\varphi d^{D} r\right)(X, Y, Z) .
\end{aligned}
$$

Here, $d \varphi \wedge h$ is defined as

$$
(d \varphi \wedge h)(X, Y, Z)=d \varphi(X) h(Y, Z)-d \varphi(Y) h(X, Z)
$$

for $h \in C^{\infty}\left(S^{2} M\right)$. From

$$
\begin{aligned}
R(X, Y, Z, W)= & \mathcal{W}(X, Y, Z, W)+\frac{1}{n-2}(g(X, Z) r(Y, W)+g(Y, W) r(X, Z) \\
& -g(Y, Z) r(X, W)-g(X, W) r(Y, Z)) \\
& -\frac{s}{(n-1)(n-2)}(g(X, Z) g(Y, W)-g(Y, Z) g(X, W)),
\end{aligned}
$$

we obtain

$$
\begin{aligned}
R(X, Y, Z, \nabla \varphi) & =\mathcal{W}(X, Y, Z, \nabla \varphi) \\
\text { (8) } \quad-\left(\frac{1}{n-2} i_{\nabla \varphi} r\right. & \left.\wedge g+\frac{1}{n-2} d \varphi \wedge r-\frac{s}{(n-1)(n-2)} d \varphi \wedge g\right)(X, Y, Z) .
\end{aligned}
$$


Here $i_{X}$ is the interior product. By combining these results, we get on $\varphi^{-1}(0)$

$$
\begin{aligned}
& \mathcal{W}(X, Y, Z, \nabla \varphi) \\
= & \left(-\frac{s}{n-2} d \varphi \wedge g+\frac{n-1}{n-2} d \varphi \wedge r+\frac{1}{n-2} i_{\nabla \varphi} r \wedge g\right)(X, Y, Z) .
\end{aligned}
$$

Therefore

$$
0=\mathcal{W}(X, Y, \nabla \varphi, \nabla \varphi)=d \varphi(X) r(Y, \nabla \varphi)-d \varphi(Y) r(X, \nabla \varphi)
$$

Thus, if $X$ is orthogonal to $\nabla \varphi$ with $Y=\nabla \varphi$,

$$
|d \varphi|^{2} r(X, \nabla \varphi)=0,
$$

implying that $z(X, \nabla \varphi)=0$ on $\varphi^{-1}(0)$.

The following lemma is needed to derive the inequality (3).

Lemma 2.2. The function $|d \varphi|^{2}+\frac{s}{n(n-1)} \varphi^{2}$ achieves its maximum value at $\Gamma$.

Proof. When $n=3$, the idea of the proof of this lemma comes from [4]. We will prove it for arbitrary dimension. Let $\Phi=|d \varphi|^{2}$. Bochner formula and the equation (7) give

$$
\begin{aligned}
\frac{1}{2} \Delta \Phi & =|D d \varphi|^{2}+\langle d \Delta \varphi, d \varphi\rangle+r(d \varphi, d \varphi) \\
& =|D d \varphi|^{2}+z(d \varphi, d \varphi)-\frac{s}{n(n-1)} \Phi \\
& =\varphi^{2}|z|^{2}+z(d \varphi, d \varphi)+\frac{s^{2}}{n(n-1)^{2}} \varphi^{2}-\frac{s}{n(n-1)} \Phi
\end{aligned}
$$

where we used the fact that $\Delta \varphi=-\frac{s}{n-1} \varphi$ and $\varphi^{2}|z|^{2}=|D d \varphi|^{2}-\frac{s^{2}}{n(n-1)^{2}} \varphi^{2}$. Making the conformal change $\tilde{g}=\varphi^{-2} g$ gives

$$
\tilde{\Delta} u=\varphi^{2} \Delta u-(n-2) \varphi\langle d \varphi, d u\rangle
$$

for any smooth function $u$ on $M$, and so

$$
\begin{aligned}
\tilde{\Delta} \Phi= & 2 \varphi^{4}|z|^{2}+2 \varphi^{2} z(d \varphi, d \varphi)+\frac{2 s^{2}}{n(n-1)^{2}} \varphi^{4}-\frac{2 s}{n(n-1)} \varphi^{2} \Phi \\
& -(n-2) \varphi\langle d \varphi, d \Phi\rangle \\
= & 2 \varphi^{4}|z|^{2}+\frac{2 s^{2}}{n(n-1)^{2}} \varphi^{4}+\frac{2(n-3)}{n(n-1)} s \varphi^{2} \Phi-2(n-3) \varphi^{2} z(d \varphi, d \varphi),
\end{aligned}
$$

since $\varphi z(d \varphi, d \varphi)=\frac{1}{2}\langle d \varphi, d \Phi\rangle+\frac{s}{n(n-1)} \varphi \Phi$. Therefore, from

$$
\tilde{\Delta} \varphi^{2}=-\frac{2 s}{n-1} \varphi^{4}-2(n-3) \varphi^{2} \Phi
$$

$$
\tilde{\Delta}\left(\Phi+\frac{s}{n(n-1)} \varphi^{2}\right)=2 \varphi^{4}|z|^{2}-2(n-3) \varphi^{2} z(d \varphi, d \varphi) .
$$


Let $F=\Phi+\frac{s}{n(n-1)} \varphi^{2}$. Then by $(10)$ and the fact that $d F=2 \varphi z(d \varphi, \cdot)$,

$$
\tilde{\Delta} F+\frac{(n-3)}{\varphi} \tilde{g}(d F, d \varphi)=2 \varphi^{4}|z|^{2} \geq 0 .
$$

Now let $M_{\varphi,-\epsilon}=\{x \in M \mid \varphi(x)<-\epsilon\}$ and $M_{\varphi}^{\epsilon}=\{x \in M \mid \varphi(x)>\epsilon\}$. By the maximum principle, $F=\Phi+\frac{s}{n(n-1)} \varphi^{2}$ does not have its maximum on the interior of the open set $M_{\varphi,-\epsilon} \cup M_{\varphi}^{\epsilon}$ for a small positive $\epsilon$. As $\epsilon$ goes to zero, we get a point $p$ in $\Gamma$ at which the function $\Phi+\frac{s}{n(n-1)} \varphi^{2}$ has its maximum value.

Proof of Theorem 1.2. The first statement of the theorem holds by Lemma 2.1. We shall prove the second statement. By Lemma 2.2, $\Phi+\frac{s}{n(n-1)} \varphi^{2}$ has its maximum value at $\Gamma=\varphi^{-1}(0)$. However, since $\Phi+\frac{s}{n(n-1)} \varphi^{2}$ is constant on each connected component of $\Gamma$, it achieves its maximum on some connected component, say $\Gamma_{0}$, of $\Gamma$.

By this observation, we obtain

$$
N_{\varphi}\left(|d \varphi|^{2}+\frac{s}{n(n-1)} \varphi^{2}\right) \leq 0
$$

on $\varphi^{-1}(\epsilon)=\partial M_{\varphi}^{\epsilon}$ for a sufficiently small positive $\epsilon$, and

$$
N_{\varphi}\left(|d \varphi|^{2}+\frac{s}{n(n-1)} \varphi^{2}\right) \geq 0
$$

on $\varphi^{-1}(-\epsilon)=\partial M_{\varphi,-\epsilon}$. Note that

$$
\begin{aligned}
N_{\varphi}\left(|d \varphi|^{2}+\frac{s}{n(n-1)} \varphi^{2}\right) & =2\left\langle D_{N_{\varphi}} d \varphi, d \varphi\right\rangle+\frac{2 s}{n(n-1)} \varphi \Phi^{\frac{1}{2}} \\
& =2 \varphi z\left(N_{\varphi}, d \varphi\right) .
\end{aligned}
$$

By combining (12), (13), and (14), we may conclude that on $\Gamma_{0}$

$$
z\left(N_{\varphi}, d \varphi\right) \leq 0,
$$

which implies the inequality (3).

The proof of Theorem 1.3 goes similarly. A non-trivial solution $(g, f)$ of $(4)$ satisfies the following equation

$$
(1+f) z=D d f+\frac{s}{n(n-1)} f g .
$$

Lemma 2.3. Let $B=f^{-1}(-1)$. The set $B$ is a union of hypersurfaces and finite points.

Proof. Let $B^{\prime}$ be the set of critical points of $f$ in $B$. Then $B \backslash B^{\prime}$ is a union of hypersurfaces. For $p \in B^{\prime}$, we have

$$
D d f_{p}(\xi, \xi)=\frac{s}{n(n-1)} g_{p}(\xi, \xi)>0
$$


for any nonzero tangent vector $\xi$ in the tangent space $T_{p} M$ at $p$. Thus $p$ is a non-degenerate critical point of $f$. Such non-degenerate critical points are isolated, and so the set $B^{\prime}$ should be finite.

Note also that $|d f|$ is constant on $B \backslash B^{\prime}$, where $B^{\prime}$ is the set of critical points in $B$ as defined in the proof of Lemma 2.3. It follows from

$$
X\left(|d f|^{2}\right)=2\left\langle D_{X} d f, d f\right\rangle=0
$$

for any tangent vector $X$ to $B$. Thus $N_{f}=d f /|d f|$ is defined on $B \backslash B^{\prime}$. Similar to Lemma 2.1, we have:

Lemma 2.4. For $p \in B \backslash B^{\prime}$ and a tangent vector $X \in T_{p}\left(B \backslash B^{\prime}\right)$ orthogonal to $\nabla f$, we have

$$
z(X, \nabla f)=0 .
$$

Proof. The proof is similar. By Ricci identity,

$$
d^{D} \operatorname{Ddf}(X, Y, Z)=R(X, Y, Z, \nabla f) .
$$

From (4),

$$
R(X, Y, Z, \nabla f)=\left(d f \wedge r+(1+f) d^{D} r-\frac{s}{n-1} d f \wedge g\right)(X, Y, Z) .
$$

Thus, by (8) we have on $f^{-1}(-1)$

$$
\begin{aligned}
& \mathcal{W}(X, Y, Z, \nabla f) \\
= & \left(-\frac{s}{n-2} d f \wedge g+\frac{n-1}{n-2} d f \wedge r+\frac{1}{n-2} i_{\nabla f} r \wedge g\right)(X, Y, Z) .
\end{aligned}
$$

In particular,

$$
0=\mathcal{W}(X, Y, \nabla f, \nabla f)=d f(X) r(Y, \nabla f)-d f(Y) r(X, \nabla f) .
$$

Hence, we may conclude that $z(X, \nabla f)=r(X, \nabla f)=0$ for $X$ orthogonal to $\nabla f$.

The following result may be compared to Lemma 2.2 for $\Gamma$.

Lemma 2.5. The function $|d f|^{2}+\frac{s}{n(n-1)} f^{2}$ achieves its maximum value at $B$.

Proof. Let $M_{f,-\epsilon}=\{x \in M \mid f(x)<-1-\epsilon\}$ and $M_{f}^{\epsilon}=\{x \in M \mid f(x)>$ $-1+\epsilon\}$. Making the conformal change $\tilde{g}=h^{-2} g$ with $h=1+f$ gives

$$
\tilde{\Delta}\left(|d f|^{2}+\frac{s}{n(n-1)} f^{2}\right)=2 h^{4}|z|^{2}-2(n-3) h^{2} z(d f, d f),
$$

since

$$
\begin{aligned}
\tilde{\Delta}|d f|^{2}= & 2 h^{4}|z|^{2}-\frac{2 s}{n(n-1)} h^{2}|d f|^{2}+\frac{2 s^{2}}{n(n-1)^{2}} f^{2} h^{2} \\
& -2(n-3) h^{2} z(d f, d f)+\frac{2(n-2)}{n(n-1)} s f h|d f|^{2}
\end{aligned}
$$


and

$$
\tilde{\Delta} f^{2}=-\frac{2 s}{n-1} h^{2} f^{2}+2|d f|^{2} h^{2}-2(n-2) f h|d f|^{2} .
$$

Thus the function $G=|d f|^{2}+\frac{s}{n(n-1)} f^{2}$ satisfies

$$
\tilde{\Delta} G+\frac{(n-3)}{h} \tilde{g}(d G, d f)=2 h^{4}|z|^{2} \geq 0 .
$$

Therefore, we can apply the maximum principle to $G=|d f|^{2}+\frac{s}{n(n-1)} f^{2}$ on the open set $M_{f,-\epsilon} \cup M_{f}^{\epsilon}$ for a small positive number $\epsilon$ to conclude that $|d f|^{2}+$ $\frac{s}{n(n-1)} f^{2}$ achieves its maximum at $B$.

We are ready to prove Theorem 1.3.

Proof of Theorem 1.3. It suffices to prove the second statement, since the first statement holds by Lemma 2.4. First we have

$$
N_{f}\left(|d f|^{2}+\frac{s}{n(n-1)} f^{2}\right)=2\left\langle D_{N_{f}} d f, d f\right\rangle+\frac{2 s}{n(n-1)} f|d f|^{2}=2 h z\left(N_{f}, d f\right) .
$$

Therefore, we may deduce that $z\left(N_{f}, N_{f}\right) \leq 0$ on some connected component of $B \backslash B^{\prime}$, similarly as in the proof of Theorem 1.2.

\section{Concluding remarks}

In this section, we shall show the connection between the behavior of the traceless Ricci tensor and the geometry and topology of the given manifold. In fact, we claim that, if the dimension of the manifold is three, then the constant scalar curvature $s_{g}$ satisfies $s_{g} \leq 24 \pi$ for both ker $s_{g}^{\prime *} \neq 0$ case and the non-trivial solution of (4) case.

Let $\left\{e_{1}, e_{2}, N_{\varphi}\right\}$ be a local orthonormal frame field near each connected component $\Gamma_{i}$ of $\Gamma$, with the normal vector field $N_{\varphi}=\Phi^{-1 / 2} d \varphi$ on $\Gamma_{i}$. Let $K_{e_{1}, e_{2}}$ be the sectional curvature of the subspace generated by $e_{1}$ and $e_{2}$, and $K_{\Gamma_{i}}$ the intrinsic Gauss curvature of $\Gamma_{i}$. Note that $K_{e_{1}, e_{2}}=\frac{s_{g}}{2}-r\left(N_{\varphi}, N_{\varphi}\right)$. Thus, by the Gauss-Codazzi equations,

$$
K_{\Gamma_{i}}=K_{e_{1}, e_{2}}=\frac{s_{g}}{2}-r\left(N_{\varphi}, N_{\varphi}\right)=\frac{s_{g}}{6}-z\left(N_{\varphi}, N_{\varphi}\right)
$$

since $\Gamma_{i}$ is totally geodesic. However, by Theorem 1.2,

$$
z\left(N_{\varphi}, N_{\varphi}\right)=\Phi^{-1 / 2} z\left(N_{\varphi}, d \varphi\right) \leq 0
$$

on some connected component $\Gamma_{0}$ of $\varphi^{-1}(0)$. Hence we may conclude that $K_{\Gamma_{0}} \geq s / 6>0$. Therefore, $\Gamma_{0}$ is homeomorphic to a 2 -sphere by Gauss-Bonnet Theorem, and

proving our claim.

$$
\frac{s_{g}}{6} \leq \int_{\Gamma_{0}} K=4 \pi
$$

Note that the last result, claiming that for $\varphi \in \operatorname{ker} s_{g}^{*}$, at least one component of the level set $\varphi^{-1}(0)$ is homeomorphic to a 2 -sphere, is already known 
([13], see also [8]). Here a lower bound on the intrinsic Gauss curvature is a new discovery.

We can argue similarly for the non-trivial solution of (4). For a local orthonormal frame field $\left\{e_{1}, e_{2}, N_{f}\right\}$ near each connected component $B_{i}$ of $B \backslash B^{\prime}$, the second fundamental form of $B_{i}$ is given by

$$
I I_{B}=|d f|^{-1} D d f=\frac{s}{6}|d f|^{-1} g .
$$

Thus the intrinsic Gauss curvature of $B_{i}$ is given by

$$
K_{B_{i}}=\frac{s}{6}-z\left(N_{f}, N_{f}\right)+\frac{s^{2}}{36|d f|^{2}} .
$$

By Theorem 1.3, $z\left(N_{f}, N_{f}\right) \leq 0$ on some connected component $B_{0}$ of $B \backslash B^{\prime}$. Thus $K_{B_{0}} \geq \frac{s_{g}}{6}+\frac{s_{g}^{2}}{36|d f|^{2}} \geq \frac{s_{g}}{6}$, which implies that $B_{0}$ is homeomorphic to a 2 -sphere, and $s_{g} \leq 24 \pi$ as in the $\operatorname{ker} s_{g}^{*} \neq 0$ case.

\section{References}

[1] S. Agmon, The $L_{p}$ approach to the Dirichlet Problem, Ann. Scuola Norm. Sup. Pisa 13 (1959), 405-448.

[2] M. Berger and D. Ebin, Some decompositions of the space of symmetric tensors on a Riemannian manifold, J. Differential Geometry 3 (1969), 379-392.

[3] A. L. Besse, Einstein Manifolds, Springer-Verlag, New York, 1987.

[4] L. Bessiéres, J. Lafontiane, and L. Rozoy, Scalar curvature and black holes, preprint.

[5] J. P. Bourguignon, Une stratification de l'espace des structures riemanniennes, Compositio Math. 30 (1975), 1-41.

[6] A. E. Fischer and J. E. Marsden, Manifolds of Riemannian metrics with prescribed scalar curvature, Bull. Amer. Math. Soc. 80 (1974), 479-484.

[7] J. Hempel, 3-manifolds, Princeton, 1976.

[8] S. Hwang, Critical points of the total scalar curvature functional on the space of metrics of constant scalar curvature, Manuscripta Math. 103 (2000), no. 2, 135-142.

[9] S. Hwang, J. Chang, and G. Yun, Rigidity of the critical point equation, Math. Nachr. 283 (2010), no. 6, 846-853.

[10] O. Kobayashi, A differential equation arising from scalar curvature function, J. Math. Soc. Japan 34 (1982), no. 4, 665-675.

[11] J. Lafontaine, Sur la géométrie d'une généralisation de l'équation différentielle d'Obata, J. Math. Pures Appl. (9) 62 (1983), no. 1, 63-72.

[12] J. Lafontaine and L. Rozoy, Courure scalaire et trous noirs, Séminaire de Théorie Spectrale et Géometrie, Vol. 18, Année 1999-2000, 69-76, Sémin. Théor. Spectr. Géom., 18, Univ. Grenoble I, Saint-Martin-d'Heres, 2000.

[13] Y. Shen, A note on Fisher-Marsden's conjecture, Proc. Amer. Math. Soc. 125 (1997), no. 3, 901-905.

Department of Mathematics

Chung-Ang University

SEOUl 156-756, KoreA

E-mail address: seungsu@cau.ac.kr 